\title{
Rapid, low-input, low-bias construction of shotgun fragment libraries by high-density in vitro transposition
}

\author{
Andrew Adey ${ }^{1 \dagger}$, Hilary G Morrison ${ }^{2+}$, Asan $^{3+}$, Xu Xun $^{3 \dagger}$, Jacob O Kitzman ${ }^{1}$, Emily H Turner ${ }^{1}$, Bethany Stackhouse ${ }^{1}$, \\ Alexandra P MacKenzie', Nicholas C Caruccio ${ }^{4}$, Xiuqing Zhang ${ }^{3^{*}}$, Jay Shendure ${ }^{1^{*}}$
}

\begin{abstract}
We characterize and extend a highly efficient method for constructing shotgun fragment libraries in which transposase catalyzes in vitro DNA fragmentation and adaptor incorporation simultaneously. We apply this method to sequencing a human genome and find that coverage biases are comparable to those of conventional protocols. We also extend its capabilities by developing protocols for sub-nanogram library construction, exome capture from 50 ng of input DNA, PCR-free and colony PCR library construction, and 96-plex sample indexing.
\end{abstract}

\section{Background}

Massively parallel DNA sequencing methods are rapidly achieving broad adoption by the life sciences research community $[1,2]$. As the productivity of these platforms continues to grow with hardware and software optimizations, the bottleneck experienced by researchers is increasingly at the front end (the construction of sequencing libraries) and at the back end (data analysis and interpretation) rather than in the sequencing itself.

The input material for commonly used platforms, such as the Illumina Genome Analyzer [3], the Roche (454) Genome Sequencer [4], the Life Technologies SOLiD platform [5], as well as for 'real-time' third-generation sequencers such as Pacific Biosciences [6], consists of complex libraries of genome- or transcriptome-derived DNA fragments flanked by platform-specific adaptors. The standard method for constructing such libraries is entirely in vitro and typically includes fragmentation of DNA (mechanical or enzymatic), end-polishing, ligation of adaptor sequences, gel-based size-selection, and PCR amplification (Figure 1a). This core protocol may be preceded by additional steps depending on the specific

\footnotetext{
* Correspondence: zhangxq@genomics.org.cn; shendure@u.washington.edu † Contributed equally

${ }^{1}$ Department of Genome Sciences, University of Washington, Seattle, WA 98195, USA

${ }^{3}$ BGI-Shenzhen, Shenzhen 518000, China

Full list of author information is available at the end of the article
}

application, such as cDNA synthesis for RNA-seq libraries [7].

Although generally effective, several aspects of the standard method are throughput-limiting or otherwise suboptimal. These include: (1) Labor: there are several labor-intensive enzymatic manipulations with obligate clean-up steps. (2) Time: the protocol requires 6-10 hours from beginning to end, often including an overnight incubation. (3) Automation: although 96-plex, semi-automated processing has been achieved by largescale genome centers [8], many researchers lack access to the requisite robotic liquid handling systems and/or instruments for parallelized mechanical fragmentation. (4) Sample indexing: incorporation of barcoded adaptors, which enable concurrent analysis of multiple samples and post-sequencing deconvolution, still requires most steps to be carried out on individual samples prior to pooling [9]. (5) High input requirements: standard protocols for shotgun DNA sequencing suggest 1-10 $\mu \mathrm{g}$ DNA as input material per library. This is often not possible, for example in cancer genomics where sample material can be limited. (6) Coverage bias: biases in sequence coverage correlated with $\mathrm{G}+\mathrm{C}$ content can arise from steps secondary to library construction, including gel purification [10] and PCR amplification [11]. Amplification-free versions of these protocols may reduce $\mathrm{G}+\mathrm{C}$ biases and eliminate PCR duplicates $[11,12]$, while potentially increasing input requirements. 


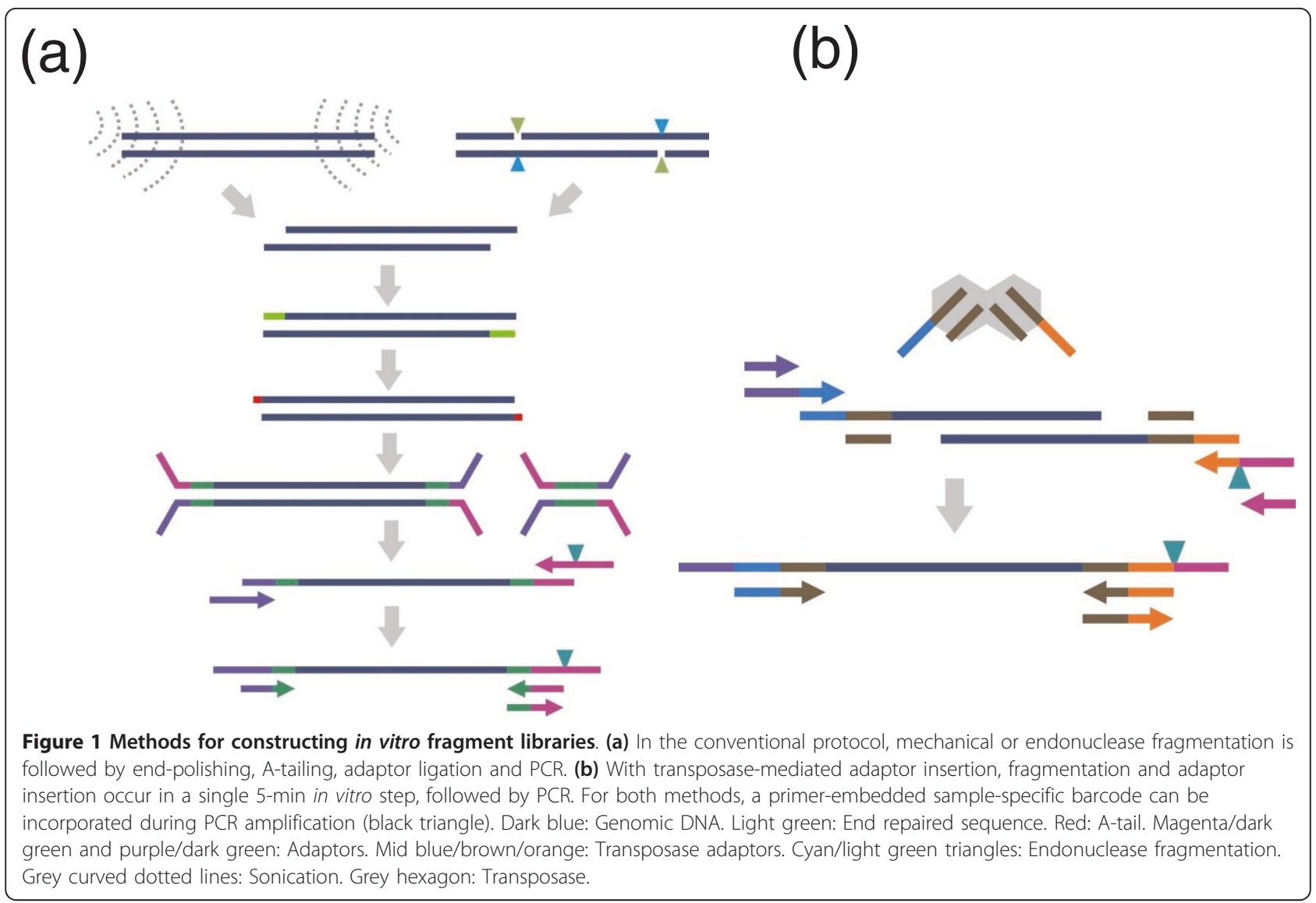

In the alternative approach that we characterize and extend here, a hyperactive derivative of the $\operatorname{Tn} 5$ transposase is used to catalyze in vitro integration of synthetic oligonucleotides into target DNA at a high density ('Nextera', Epicentre, Madison, WI, USA). Wild-type Tn5 transposon DNA is flanked by two inverted IS50 elements, each containing two 19 bp sequences required for function (outside end and inside end). A $19 \mathrm{bp}$ hyperactive derivative (mosaic end, ME) is sufficient for transposition provided that the intervening DNA is long enough to allow the two ends to come in close proximity in order to form a complex with a Tn5 transposase homodimer. The relatively low activity of the wild-type Tn5 transposase was cumulatively increased through several classes of mutation [13]. In a classical in vitro transposition reaction, hyperactive Tn 5 transposomes (hyperactive transposase mutant bound to ME-flanked DNA) bind target DNA and catalyze the insertion of ME-flanked DNA into the target DNA with high frequency [14]. When free synthetic ME adaptors are used instead (isolated from one another, in contrast to MEflanked DNA in which two ME sequences are linked by the intervening DNA), transposase activity results in fragmentation and end-joining of the synthetic ME adaptor to the 5' end of target DNA. To generate fragment libraries compatible with massively parallel DNA sequencing, limited-cycle PCR is used to append platform-specific primers (Figure 1b).

Significant potential advantages of transposase-catalyzed adaptor insertion as a library preparation method, relative to conventional library preparation, include, firstly, many fewer steps, as the fragmentation, polishing, and ligation steps are replaced by a single 5 -minute reaction and optional 10-minute pre-PCR clean-up (Figure 2). Libraries requiring particularly constrained insert size distributions (such as for de novo assembly) may optionally be subjected to chipor gel-based size selection, increasing preparation time by 1 hour or 3-4 hours, respectively. The second advantage is greatly reduced input requirements while maintaining library complexity. This is expected to be possible because of a more efficient conversion of input DNA into sequencing-compatible material. However, these potential advantages are balanced by the competing concern that transposase-mediated fragmentation will introduce significant sequencedependent biases relative to conventional library construction. 


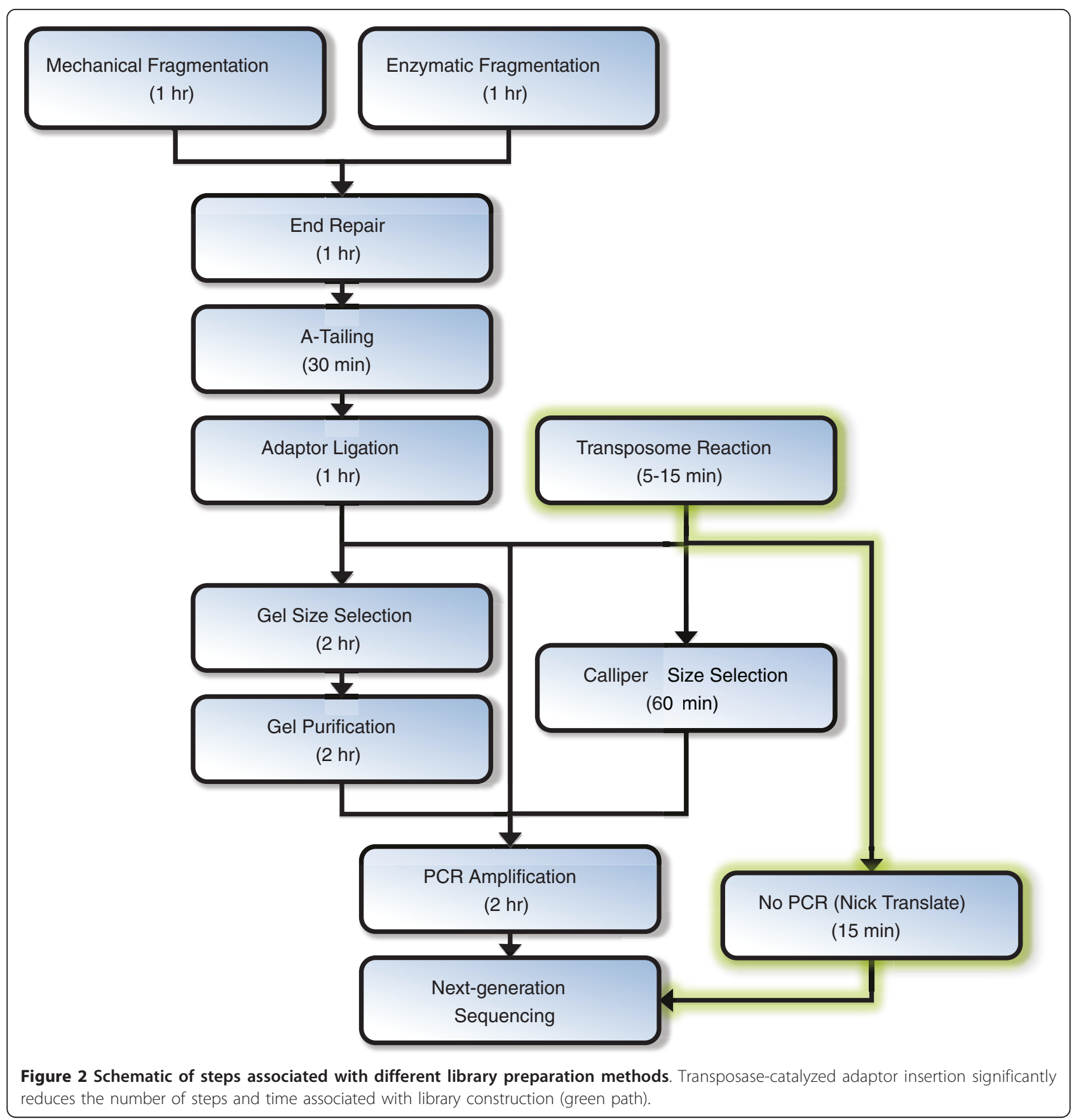

Here, we report the results of an extensive comparison of transposase-catalyzed fragmentation with standard library construction protocols. We also describe the development of several derivative protocols for transposase-catalyzed fragmentation that significantly extend its capabilities. To evaluate performance with respect to key parameters including sequence-dependent biases, we compared methods across several organisms and sequencing platforms, including whole genome sequencing of a cell line derived from a previously sequenced human, YH1 [15], on a single flow-cell with the Illumina HiSeq platform. New protocols reported here that extend the utility of this method include: (1) a 96-plex sample indexing scheme, validated on 96 bacterial genomes; (2) capture and sequencing of the complete coding exon content (exome) from $50 \mathrm{ng}$ of input human genomic DNA; (3) a protocol for the construction and sequencing of shotgun libraries from as little as $10 \mathrm{pg}$ of starting material; (4) a PCR-free version of the method that mitigates associated $\mathrm{G}+\mathrm{C}$ biases and decreases the 
total time for library preparation time to less than 30 minutes; and (5) a method analogous to 'colony PCR' for single-step preparation of genomic sequencing libraries directly from bacterial colonies.

\section{Results \\ Comparison of standard versus transposase-based protocols}

We performed a side-by-side comparison of three protocols: (1) standard library construction with mechanical fragmentation; (2) standard library construction with time-dependent endonuclease-based fragmentation ('dsDNA fragmentase', NEB); and (3) transposasecatalyzed adaptor insertion ('Nextera', Epicentre). To evaluate performance on the Illumina platform, sequencing libraries and technical replicates were prepared from two genomic DNA samples (Homo sapiens NA18507, Escherichia coli CC118) with each of the three methods. Paired-end, 36 bp reads were generated on an Illumina Genome Analyzer IIx (GAIIx). Reads were mapped using BWA [16] to the E. coli genome (K12) or human genome (hg18) as appropriate. To evaluate performance on the Roche (454) platform, sequencing libraries were constructed from two bacteriophage DNAs (CRW10 and PA1) with each of the three methods. Libraries were sequenced on a Roche (454) Genome Sequencer FLX, followed by de novo assembly (gsAssembler) and read mapping (gsMapper) to the appropriate reference genome. A summary of samples processed and sequence data generated on both platforms is provided in Table S1 in Additional file 1.

Sites of mechanical fragmentation, endonuclease fragmentation, and transposase-catalyzed adaptor insertion were characterized by calculating nucleotide composition in the vicinity of the mapping position of the first base of each sequence read (the fragmentation site; Figure S1 in Additional file 2). This revealed a slight but highly correlated bias for mechanical and endonuclease fragmentation, which suggests that most bias for these two methods is introduced after these protocols converge (for example with A-tailing or adaptor ligation), and that both mechanical fragmentation (here, either acoustic sonication or nebulization) and endonuclease fragmentation (with dsDNA fragmentase) have very low intrinsic biases. In contrast, a more extended signature is observed for sites of transposase-catalyzed adaptor insertion, weakly resembling the reported insertion preference of the native Tn5 transposase (AGNTYWRANCT, where $\mathrm{N}$ is any nucleotide, $\mathrm{R}$ is $\mathrm{A}$ or $\mathrm{G}, \mathrm{W}$ is $\mathrm{A}$ or $\mathrm{T}$, and $\mathrm{Y}$ is $\mathrm{C}$ or $\mathrm{T}$ ) [17]. However, when calculated in terms of per-position information content, the bias of transposase-catalyzed adaptor insertion is low, and only slightly greater than the other protocols. For E. coli data, maxima of per-position information content over $\pm 10 \mathrm{bp}$, on a two-bit scale for fixed positions, are $0.10,0.11$, and 0.16 for mechanical fragmentation, endonuclease fragmentation, and transposase-catalyzed adaptor insertion, respectively. Average information content over \pm 10 bp are $0.0056,0.018$, and 0.049 , respectively. Equivalently low information contents were observed for human and phage libraries (Table S2 in Additional file 1). The effective bias associated with transposasecatalyzed adaptor insertion is thus greater than with standard library construction, but only modestly so. For $E$. coli and human libraries, signatures of bias were consistent in technical replicates for all three methods.

The greater insertion bias is problematic in a practical sense only if it has a significant impact on the distribution of genomic coverage. Consistent with the low calculated information content of the observed biases, the gross distributions of genomic coverage observed for the three methods are very similar (Figure 3a, b), the exception being the PA1 bacteriophage library, which may be skewed as a result of sequence context in a relatively small genome. Furthermore, similar biases in coverage are observed for different $\mathrm{G}+\mathrm{C}$ content bins, with reduced representation at both extremes (Figure 3c). As PCR was used to prepare libraries constructed with all three methods, the consistent $\mathrm{G}+\mathrm{C}$ bias probably arises at that step [11]. We initially predicted that the similar genomic coverage distribution associated with each method was due to factors introduced after the three protocols converge on common steps (solution phase PCR, cluster PCR, and sequencing). However, the correlation in coverage between methods at a per-base level was modest, with transposase-catalyzed adaptor insertion the least correlated with the other methods (Table S3 in Additional file 1).

In this comparative analysis, libraries generated by transposase-catalyzed adaptor insertion were sequenced directly after PCR (without size-selection), and the observed insert size distribution was considerably shorter than the other, size-selected, methods (transposase: $100 \pm 47 \mathrm{bp}$, sonication: $256 \pm 48 \mathrm{bp}$, endonuclease: $244 \pm 56$ bp; Figure S2 in Additional file 2). To evaluate whether a lower-bound on insert size exists, tails of long-read (101 bp) pairs were aligned to one another and a mapping-independent size distribution constructed, revealing a sharp decrease at about $35 \mathrm{bp}$ that is probably a secondary consequence of steric hindrance of adjacent, attacking transposases (Figure 4). This phenomenon also explains the about 10 bp peaks at the lower end of the insert size distribution resulting from the helical pitch of the DNA as it extends away from the transposase.

With alternative buffer and reaction conditions, other target size ranges can be achieved. For example, the transposon method adapted for Roche (454) library 

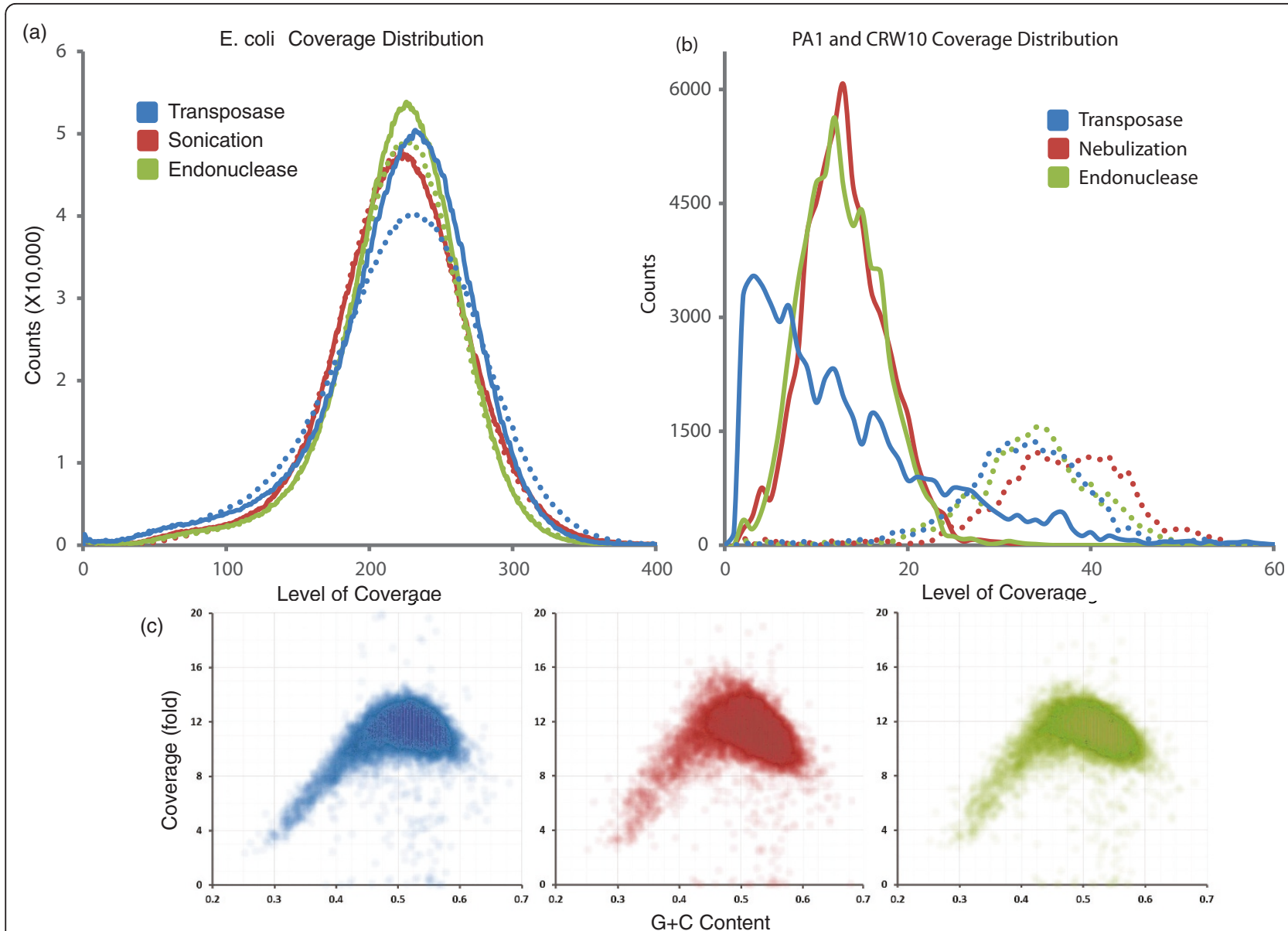

Figure 3 Comparison of coverage bias. (a) Coverage distribution across the E. coli genome with transposase (blue), sonication (red), and endonuclease (green) methods (solid lines) and replicates (dotted lines), normalized for total sequencing depth. (b) Coverage distribution across the PA1 and CRW10 bacteriophage genomes with transposase (blue), nebulization (red), and endonuclease (green) methods (dotted lines represent replicate libraries). (c) $\mathrm{G}+\mathrm{C}$ bias for $E$. coli was assessed by calculating $\mathrm{G}+\mathrm{C}$ content of the reference in 500 bp bins and plotting the coverage in each for transposase (blue), sonication (red), and endonuclease (green) methods, all of which show an approximately equivalent bias against the extremes.

construction resulted in significantly longer fragments (300-800 bp; Figure S3 in Additional file 2). To assess whether fragment size of libraries generated by transposase-catalyzed adaptor insertion could be constrained without resorting to gel-based size-selection, we evaluated alternative buffer and reaction conditions in combination with different approaches to post-PCR sample clean-up (Figure S4 in Additional file 2). Notably, an automated chip-based size-selection yielded well-constrained libraries (insert size $162 \pm 28 \mathrm{bp}$ ).

\section{Whole genome sequencing of human and Drosophila genomes}

To assess performance further, we conducted whole genome sequencing on transposase-based libraries from H. sapiens and Drosophila melanogaster. Human genomic DNA from a previously sequenced individual, YH1 [15], was used to generate a series of libraries under different reaction conditions and size-selections that were then subjected to seven lanes of paired-end 90 bp (PE90) sequencing on the Illumina HiSeq platform. Of 934 million reads, 781 million were mapped [16] to the human genome (hg18) for $25 \times$ coverage. Although a total of seven libraries were constructed and sequenced to assess reproducibility, the complexity of each individual library was sufficient enough that whole genome sequencing could be carried out using a single library. Variant calling on mapped YH1 data was performed with samtools [18] requiring consensus Q30 at called positions (Figure S5 in Additional file 2). By these criteria, 3,556,679 SNPs were called ( $87 \%$ in dbSNP129; transition/transversion ratio $(\mathrm{Ti} / \mathrm{Tv})=2.07)$, substantially greater than the 3,074,097 SNPs reported in initial sequencing of YH1. There were 2,922,525 SNPs shared between the analyses (91\% in dbSNP129; Ti/Tv = 2.07), 634,154 SNPs unique to our analysis of this genome $(70 \%$ in dbSNP129; $\mathrm{Ti} / \mathrm{Tv}=2.08)$, and $151,572 \mathrm{SNPs}$ 


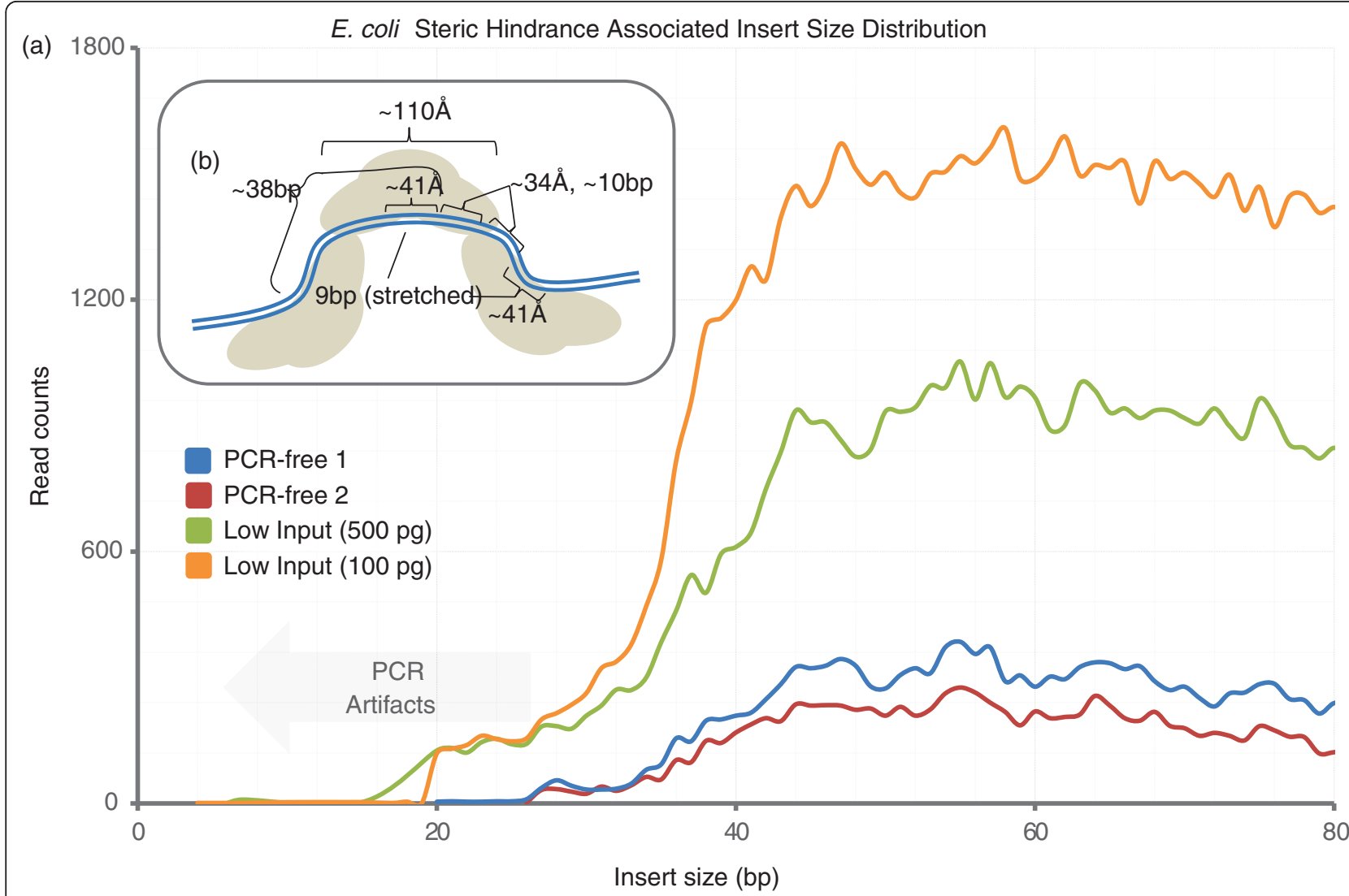

Figure 4 Insert size showing steric hindrance. (a) Insert size was generated from libraries spiked into a paired-end 101 bp run resulting in a large proportion of reads reading into the adaptor sequence. Tails of reads were then aligned to one another to discern the insert size between adaptors, resulting in a mapping-independent insert size at the lower extreme. All reads with an insert size less than 25 bp were PCR artifacts. (b) The noticeable drop below $40 \mathrm{bp}$ is consistent with a model for complete saturation of transposition events on a given stretch of DNA. The roughly $110 \AA$ transposase homodimer (grey) is bound to genomic DNA (blue), such that the core of the enzyme acts on a 9 bp region drawn out to $41 \AA$ as well as approximately 10 additional bases of DNA flanking either side ( $\sim 34 \AA$ each) that are essentially protected from a subsequent transposase attack due to steric hindrance. Since the core region is duplicated in the process, the minimum spacing of transposition events is approximately $38 \mathrm{bp}$.

unique to the initial analysis of this genome $(65 \%$ in dbSNP129; $\mathrm{Ti} / \mathrm{Tv}=1.18)$. The larger number of SNPs identified here may follow in part from greater mappability with longer read-lengths.

In this analysis, cell-line DNA derived from lymphoblasts was used; however, original sequencing of $\mathrm{YH} 1$ by Wang et al. (2008) [15] was carried out on blood DNA. Notably, 4,036 positions were called as mutations in the cell line and as the reference base in blood, both at a high quality score (30) and in uniquely mappable regions of the genome (see Methods). Of the 1,720 SNPs at a quality over 50 $(\mathrm{Ti} / \mathrm{Tv}=0.95)$, a randomly selected 100 were subjected to validation in DNA from blood, DNA from the primary culture used to generate the cell line, and DNA from the cell line. Interestingly, 63 were confirmed as mutations only in the cell line $(\mathrm{Ti} / \mathrm{Tv}=1.1$; one failed assay in primary culture). Of the 37 positions that failed validation, 31 were confirmed as the reference base in blood, primary culture, and cell line $(\mathrm{Ti} / \mathrm{Tv}=0.48)$, and the remaining six positions were variant in all three $(\mathrm{Ti} / \mathrm{Tv}=1.0$; one failed assay in primary cell culture). Further experimentation is required to determine whether the validated mutations observed in the cell line represent only mutations occurring during immortalization or propagation of the cell line, or the eventual fixation of somatic mutations present at very low frequencies in primary culture.

Importantly, coverage of YH1's genome in sequencing of libraries derived from transposase-catalyzed fragmentation was relatively uniform when compared with the data generated on this same individual from conventional libraries (Figure 5a). The observed GC bias in whole human genome sequencing data from the two methods was comparable (Figure 5c); however, a modest decrease $(23 \%)$ in coverage at bins with high GC content $(\geq 60 \%)$ was observed with the transposase method. This decrease can potentially be mitigated by a PCR-free version of this 


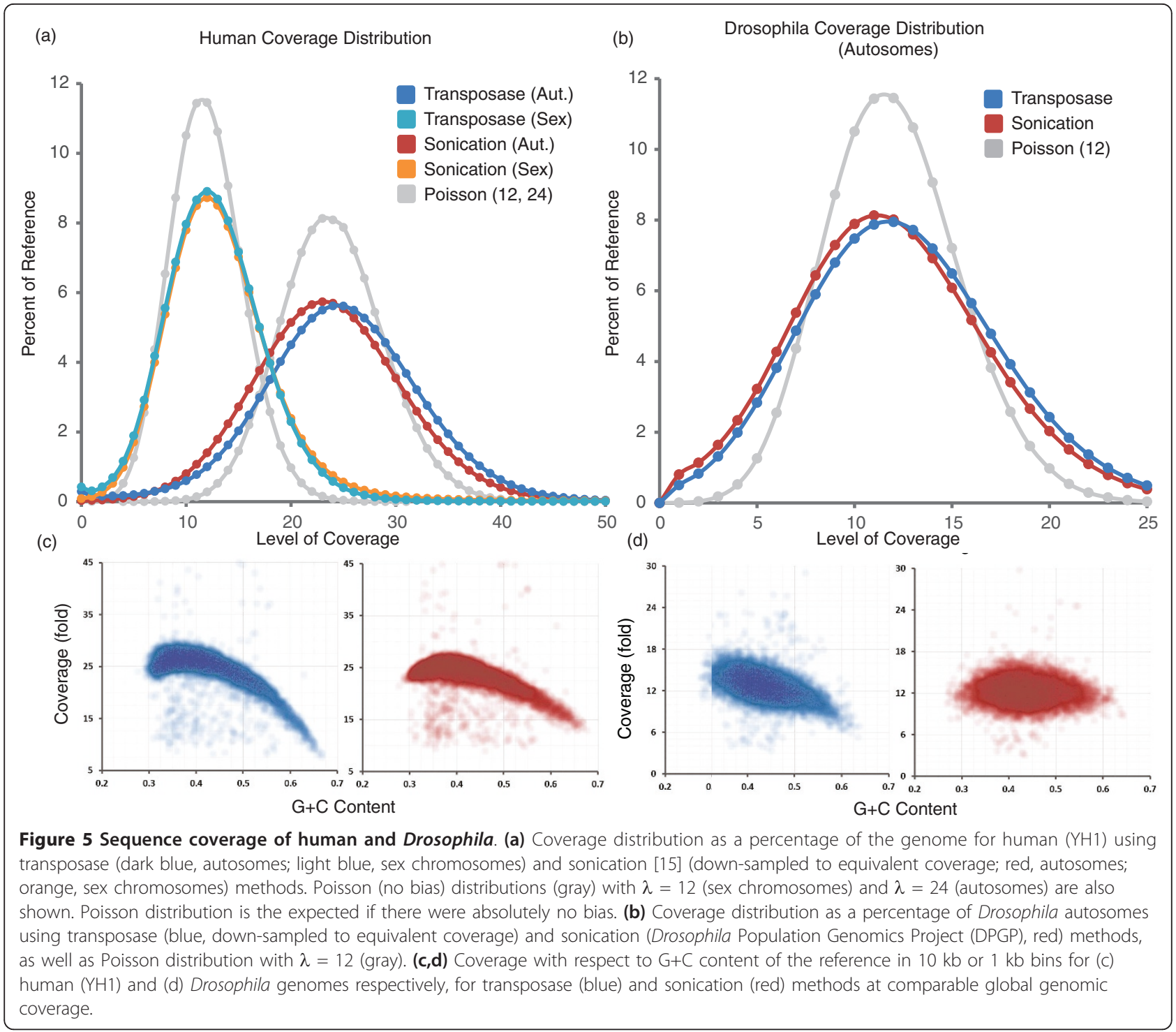

method (discussed below), or by alternative PCR conditions (data not shown). For the Drosophila genome (dm3), one lane of PE45 sequencing of a transposase-based library on an Illumina GAIIx yielded $16 \times$ mean coverage. As with the human genome, the distribution of coverage was largely equivalent to that observed in sequencing Drosophila with standard libraries (Figure 5b, d) along with the modest decrease in coverage for regions of high GC content. For both human and Drosophila genomes, the signature of bias in the vicinity of insertion sites was similar to that observed in the comparative analysis.

Complexity, that is, the number of molecules of distinct origin, is a critical aspect of shotgun library quality, especially for libraries that will be deeply sampled or subjected to further bottlenecking, such as hybrid capture or size selection. Low complexity manifests as an excess of duplicate reads with identical mapping coordinates, which arise from the same progenitor molecule and can thus skew downstream analyses including SNP calling and genotyping. The complexity of each prepared library here was analyzed by incremental sampling of 50,000 read-pairs without replacement and plotting the number of read-pairs sampled versus the number of unique read-pairs (as determined by mapping location) within the sample. In this analysis, the extent of deviation from linearity provides a measurement of sample complexity. All human genome and Drosophila genome libraries sequenced here were found to be highly complex, each comprising over $96 \%$ unique read-pairs, even as sequencing depths approached 100 million read-pairs, indicating a single such library could be used for whole genome sequencing (Figure 6). The high complexities achieved are consistent with a high efficiency of conversion of input mass into sequence- 


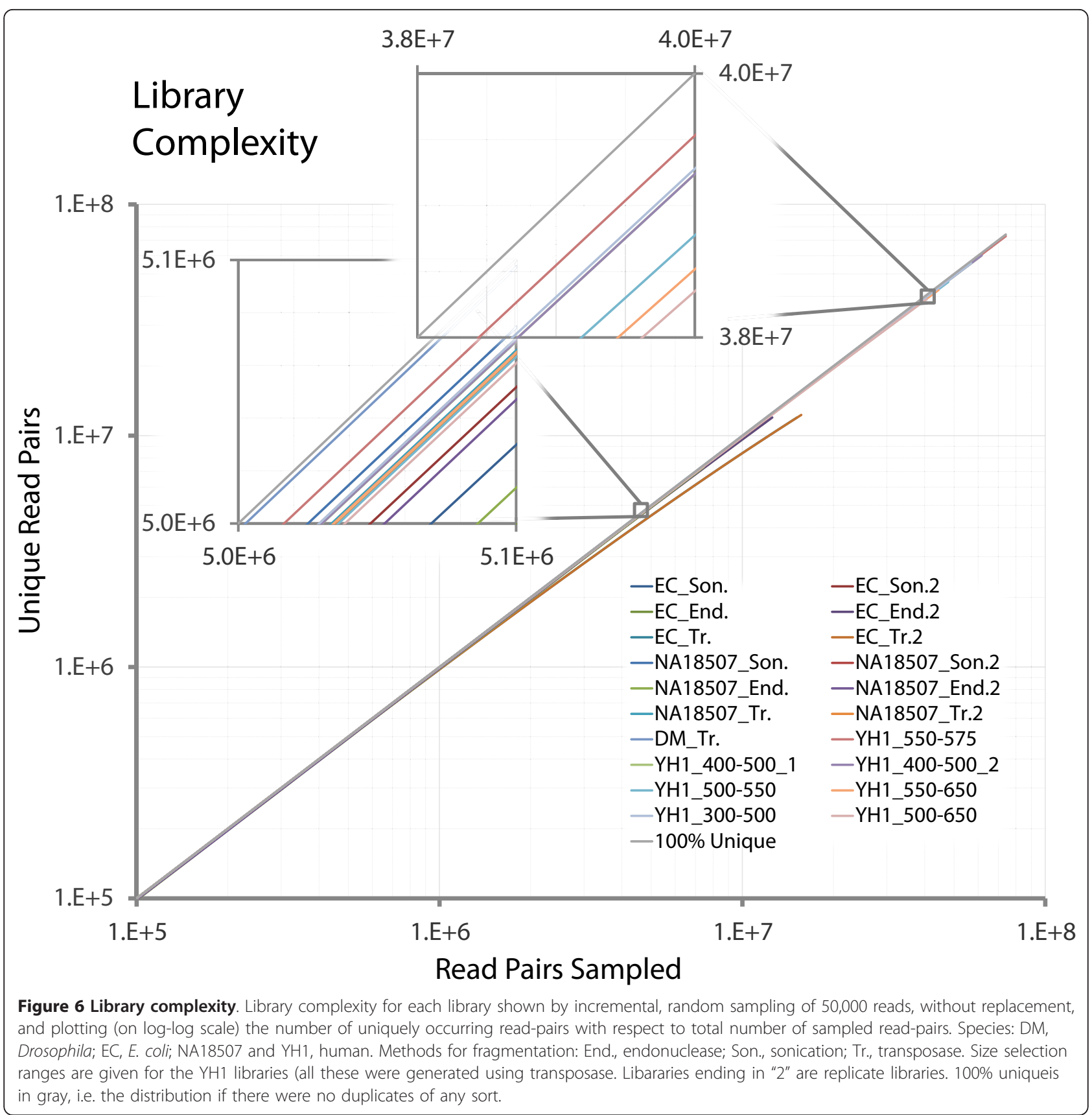

compatible material as compared with conventional library construction. Less complexity was consistently observed for all E. coli libraries, but this is likely to be because we are simply saturating the set of possibilities for start-point pairs that are used to identify PCR duplicates by deep sequencing of a small genome.

\section{Low input targeted sequence capture of the} human exome

Exome capture is an increasingly mature technology, but standard protocols require several micrograms of input genomic DNA, which can be problematic when sample is limiting (for example with tumor samples). We subjected a library from $50 \mathrm{ng}$ human genomic DNA by transposome fragmentation to exome capture (Nimblegen SeqCap EZ Exome probes v1.0). Because the adaptor sequences are different from those in libraries prepared using mechanical shearing, custom blocking oligonucleotides were designed and used. After capture, the library was subjected to pre-sequencing real-time PCR with standard primers followed by sequencing on an Illumina GAIIx (SE36). The resulting reads were 
aligned to the human genome (hg18) with $78 \%$ mapping, of which $47 \%$ fell within $100 \mathrm{bp}$ of a targeted exon (Figure S6 in Additional file 2). A direct comparison with an equivalent number of mapped SE36 reads from a standard library, after capture with the same kit, revealed nearly identical complexity for on-target reads (41\% and $43 \%$ of an equivalent number of on-target SE36 reads with unique start sites for transposomebased and standard libraries, respectively), as well as comparable uniformity ( $87 \%$ and $82 \%$ of target bases covered with $\geq 1$ reads for transposome-based and standard libraries, respectively). However, specificity was notably lower ( $47 \%$ of reads on or near target for transposome-based libraries versus $80 \%$ for standard libraries). Nonetheless, we note that the standard protocol has been extensively optimized in the context of production-level scaling, and it is likely that specificity in the capture of transposome-based libraries can also be improved upon. Furthermore, the disadvantage of lower specificity is balanced by the advantage of significantly lower input requirements for genomic DNA entering a targeted capture workflow (50 ng for transposase-based libraries versus $3 \mu \mathrm{g}$ for standard libraries).

\section{Sub-nanogram library construction}

To push the limits on library construction using reduced starting material, E. coli libraries were generated from $500 \mathrm{pg}$ and $100 \mathrm{pg}$ genomic DNA and sequenced as part of a barcode pool. For each library, expected numbers of read counts were observed ( 0.5 and 0.6 million mapped reads, respectively) without a noticeable drop in complexity (both libraries over $98 \%$ at 0.5 million read-pairs), or coverage uniformity. Next, we generated a library from 10 pg human genomic DNA, or roughly three copies of the human genome, which produced over
2 million uniquely mapped read-pairs. Although complexity was reduced because of the significant decrease in progenitor molecules entering PCR, the potential advantages of sequencing from material approaching a single equivalent of the human genome are substantial.

\section{PCR-free library construction}

Standard sequencing libraries for the Illumina platform have been generated without the use of PCR amplification in order to reduce associated biases [11,12]. We developed a similar approach for transposase-based methods by including sequences corresponding to the primers used for cluster formation, i.e. the Illumina adaptor sequences, into the adaptors that are added during the transposition reaction, as opposed to incorporating them during PCR (See Methods). After transposition, a nick translation is performed resulting in Illumina-ready libraries. This method was used to sequence E. coli CC118 and human NA18507 with two replicates of each using $100 \mathrm{ng}$ and 200 ng starting material. A noticeable decrease in $\mathrm{G}+\mathrm{C}$ coverage bias was observed (Figure 7a). Furthermore, complexity for each of these libraries was over $98 \%$. The development of PCR-free, transposase-based library construction reduces the full amount of time required for converting DNA to a sequencing-ready shotgun library to less than 30 minutes.

\section{6-plex sample indexing}

Second-generation sequencing platforms suffer from poor granularity. For example, in the data described above, a single PE90 lane on the Illumina HiSeq platform yielded $20 \mathrm{~Gb}$ of mappable sequence, which is far in excess of what is required for many projects. Sample indexing (or 'barcoding') is a useful solution, but reported protocols still require most steps of library
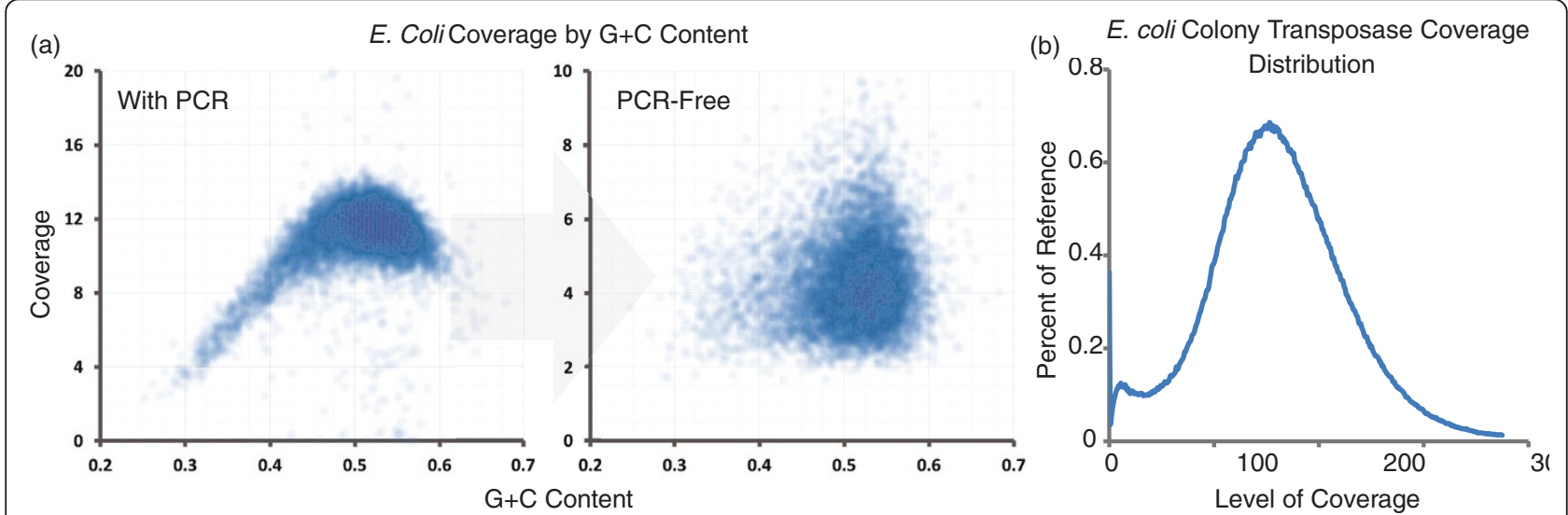

Figure 7 PCR-free reduction in $\mathrm{G}+\mathrm{C}$ coverage bias and direct-from-colony coverage distribution. (a) Coverage with respect to $\mathrm{G}+\mathrm{C}$ content in $E$. coli with and without PCR was assessed by calculating $\mathrm{G}+\mathrm{C}$ content of the reference in $500 \mathrm{bp}$ bins and plotting the coverage in each for transposase after PCR (left) and with the PCR-free method (right). A significant reduction in coverage bias at the extremes of $\mathrm{G}+\mathrm{C}$ content is observed. (b) Coverage distribution for E. coli library prepared directly from cell lysate without purification. 
preparation to be carried out on individual samples prior to pooling, and also can suffer from non-uniform performance of individual barcodes [9]. With transposase-catalyzed adaptor insertion, sample indexing could potentially be introduced during adaptor insertion, or during the subsequent PCR step, that is, using a primerembedded barcode sequence (Figure 1b). To evaluate the compatibility of this method with indexing, we attempted the latter approach. Ninety-six barcodes (9 bp) were designed with a minimal edit distance of four between all pairs and additional constraints on base composition (Table S4 in Additional file 3). Performance was evaluated by subjecting DNA from 96 evolved derivatives of Pseudomonas aeruginosa to independent library construction, each with a different barcodeembedded primer during PCR. Post-PCR amplicons were quantified and pooled, followed by several lanes of massively parallel sequencing (PE76, with a third read to collect the $9 \mathrm{bp}$ index). Samples were deconvolved using 9 bp indexes: $92 \%, 3 \%$, and 3\% reads were assigned with 0,1 , and 2 mismatches, respectively, and only $4 \%$ could not be unambiguously assigned. With the exception of a few outliers, the distribution of barcode assignments across the 96 was relatively uniform with $90 \%$ within a fourfold range (Figure S7 in Additional file 2), as was the proportion of reads mapping to the reference, illustrating the robustness of the library protocol and the indexing scheme.

\section{Constructing genomic libraries directly from bacterial colonies}

In evaluating 96-plex sample indexing for 96-plex bacterial genome sequencing with transposase-catalyzed adaptor insertion, the burden of technical effort shifted from library preparation to the isolation of genomic DNA from each isolate. We speculated that integration of the transposase reaction into a 'colony PCR'-like workflow (in which cells from bacterial colonies are directly mixed into a PCR reaction without DNA isolation) could be used to further simplify library preparation for bacterial genome sequencing (Figure S8 in Additional file 2). A pipet tip was used to transfer a small number of cells directly from an E. coli colony transformed with pUC19 to a transposase fragmentation reaction (with heat-lysing prior to addition of enzyme). An aliquot of this reaction served as input for PCR amplification without any intervening clean-up step. Sequencing (SE36) yielded 27 million reads, for $170 \times$ coverage of the E. coli genome (81\% of reads) and $37,000 \times$ coverage of pUC19 (10\% of reads). The remaining $8 \%$ mapped to the $\mathrm{F}$ plasmid, or an insert cloned into pUC19, or remained unmapped. Coverage of the E. coli genome was uniform (Figure $7 \mathrm{~b}$ ). We propose that direct preparation of genomic sequencing libraries from bacterial colonies with no DNA isolation or intervening purification steps will be useful for rapidly preparing sequencing-ready, indexed fragment libraries from large numbers of bacterial isolates.

\section{Discussion}

Massively parallel sequencing platforms generally require the conversion of genomic DNA (or other nucleic acid sample) into a fragment library that includes common adaptor sequences that are used to mediate clonal amplification and/or the priming of sequencing reactions. Practical limitations of conventional approaches to generating these libraries include high requirements for labor, time and cost, as well as the low efficiency of mass conversion into sequencingcompatible material. Here, we evaluate an alternative approach in which transposase catalyzes the fragmentation of target DNA and insertion of adaptor sequences in a 5-minute, small-volume reaction. The workflow is thus markedly simpler than the conventional approach yielding significant savings in terms of time and labor (summarized in Figure 2). The input requirements are also over an order of magnitude lower than what is typically used with standard methods, and we demonstrate that high complexity libraries can be generated from as little as $100 \mathrm{pg}$ of input DNA. Furthermore, input can be reduced to as low as just a few copies of the human genome and still produce a significant amount of sequence data. Taking advantage of the simplicity and low input requirements, we developed a method to construct libraries directly from bacterial colonies without DNA isolation or intervening clean-up steps.

Although there are significant advantages, this method nonetheless has its limitations. First, although there is a significant reduction in required steps and time, preparing very large numbers of libraries is still challenging without some degree of automation. Second, one has relatively limited control over the size distribution of fragmentation. In general, the trend is that the insert size distribution is smaller than desired when reacting to completion, and broader than desired when altering reaction conditions to increase the mean insert size. Third, genomes with high $\mathrm{G}+\mathrm{C}$ contents show greater bias with this method than with conventional methods, although this is potentially correctable in part by the PCR-free approach or through modified PCR conditions. A related point is that we have also observed that performing transposase-catalyzed adaptor insertion on a single PCR product results in significantly greater bias than with shotgun libraries (J. Hiatt, personal communication), potentially secondary to a high molar concentration of a limited number of possible insertion sites. Fourth, we note that this library preparation does not solve an ongoing challenge in the field, which is how 
best to efficiently construct high-complexity, long-insert mate-paired libraries.

A comparison of sequence composition in the vicinity of fragmentation sites identified a bias signature that was larger and more extended than that associated with conventional methods. However, we found that this had little impact at the level of coverage for bacterial, human, and Drosophila genomes. Nonetheless, the observed impact may be greater in genomes with compositions that correlate with this bias pattern, or in smaller genomes such as bacteriophage. In our view, for most goals, the disadvantage of the slightly greater bias is offset by the large advantages that we observed with respect to speed, simplicity, and low input requirements.

A further reduction in preparation time was achieved in developing a PCR-free, transposase-based library construction method. This approach also decreased biases resulting from amplification, notably in coverage with respect to $\mathrm{G}+\mathrm{C}$ content. Looking ahead, we anticipate that the partnership of extremely fast, simplified methods for library construction with third-generation 'realtime' sequencing methods may represent a critical path forward in reducing the time between acquiring biological material and obtaining analyzed sequence data to less than 1 hour.

\section{Conclusions}

Current methods for preparing in vitro DNA fragment libraries for massively parallel sequencing are suboptimal for projects involving limited amounts of starting material or large numbers of samples. Here we have characterized an alternative method of library construction in which highly active transposase catalyzes the simultaneous fragmentation and adaptor ligation in a single 5-minute incubation. Comparison with conventional methods of library preparation, relying on mechanical or endonuclease fragmentation, finds that although transposase-catalyzed adaptor insertion demonstrates a slightly greater insertion bias, this has little impact at the level of genomic coverage and is offset by large advantages with respect to speed, simplicity, and low input requirements.

To fully take advantage of the method and expand on transposase-catalyzed adaptor insertion we applied it on a larger scale, including sequencing a human genome on a single flow-cell of a massively parallel sequencing platform, and validating a 96-plex sample indexing scheme. We also show that transposase-catalyzed adaptor insertion can be integrated with exome sequencing workflows, with the advantage of significantly lower input requirements relative to conventional protocols for targeted sequence capture (50 ng versus several micrograms). In addition, we have generated libraries from as little as $10 \mathrm{pg}$ of starting material and also developed a PCR-free library construction method in order to reduce associated biases and further reduce preparation time to less than 30 minutes. Finally, we demonstrate the direct preparation of genomic sequencing libraries from bacterial colonies with no DNA isolation or intervening purification steps.

\section{Materials and methods \\ Genomic DNA}

Genomic DNA for H. sapiens NA18507 (Yoruban) was prepared by the Coriell Institute. E. coli CC118

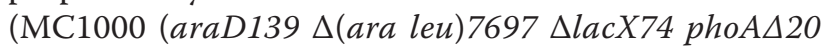
galE galK thi rpsE rpoB argE $E_{\text {am }}$ recA1)) [19], genomic DNA, and CRW10 and PA1 phage genomic DNA were extracted using Qiagen (Valencia, CA, USA) MidiPrep buffers P1, P2, P3 and were cleaned by phenol, purified from low melting point agarose and dissolved in Tris/ EDTA (TE) buffer. YH1 genomic DNA was extracted from a lymphoblastoid cell line of Yanhuang [15] using protein $\mathrm{K}$ and phenol/chloroform [20] and further subjected to RNase treatment/purification. The D. melanogaster genomic DNA was extracted from whole bodies of several individuals by Puregene blood core kit B (Qiagen). The molecular weights of both Drosophila and YH1 genomic DNAs were confirmed to be larger than $23 \mathrm{~kb}$ by gel electrophoresis (not shown), with no detection of degradation or RNA/protein contamination, and quantified by Quant-iT dsDNA HS assay kit (0.2-100 ng; Invitrogen, Q32854, Carlsbad, CA, USA); of each, an aliquot was diluted to $25 \mathrm{ng} / \mu \mathrm{l}$ for use in library construction. $P$. aeruginosa PAO1 strains were selected for tobramycin resistance at $16 \mathrm{mg} / \mathrm{l}$ (41 strains), ciprofloxacin resistance at $4 \mathrm{mg} / \mathrm{l}$ (47 strains), or no antibiotic resistance (8 strains). DNA was then isolated using the Wizard $^{\circledR}$ SV 96 genomic DNA purification system (Promega, Madison, WI, USA). Concentrations of isolated DNA were measured using a Nanodrop (Thermo Scientific, Waltham, MA, USA).

\section{Mechanical shearing}

Shearing was performed on two $5-\mu \mathrm{g}$ aliquots of E. coli CC118 genomic DNA brought up to $200 \mu$ with TE. Sonication was performed by two 15-min treatments with a Bioruptor sonicator (Wolf Laboratories, Pocklington, UK) at maximum settings in a cold room, switching out the water between treatments to keep samples cool. After sonication, each sample was cleaned up using a QIAquick PCR purification kit, eluting in $30 \mu \mathrm{l}$ Buffer EB (Qiagen). CRW10 and PA1 phage DNA was fragmented using $5 \mu \mathrm{g}$ aliquots brought to $100 \mu \mathrm{l}$ with TE and mixed with $500 \mu \mathrm{l}$ nebulization buffer (Roche/454, Branford, CT, USA) in a nebulizer cup. Nebulization was carried out using $45 \mathrm{psi}(310 \mathrm{kPa})$ nitrogen for 1 min on ice. The sheared DNA was cleaned and 
concentrated using MinElute columns and eluting in Buffer EB (Qiagen).

\section{Enzymatic fragmentation}

Enzymatic fragmentation was performed on six $1 \mu \mathrm{g}$ aliquots of genomic DNA from both E. coli CC118 and $H$. sapiens NA18507 added to $2 \mu \mathrm{l} 10 \times$ fragmentation reaction buffer, $0.2 \mu \mathrm{l} 100 \times \mathrm{BSA}$ (NEBiolabs, Ipswich, MA, USA), $2 \mu$ NEB fragmentase enzyme, and nuclease-free water (Ambion, Austin, TX, USA) to $20 \mu \mathrm{l}$. Reactions were gently vortexed and spun-down, then incubated on ice for $5 \mathrm{~min}$ followed by a time-course incubation at $37^{\circ} \mathrm{C}$, removing samples at $15,20,30,45$, 60 , and $120 \mathrm{~min}$ for each of the two sets. Reactions were stopped by placing on ice followed by purification using a QIAquick PCR purification kit, eluting in $30 \mu \mathrm{l}$ buffer EB (Qiagen). $4 \mu \mathrm{l}$ of each sample was run on a Novex TBE gel (Invitrogen) to observe size distribution. The 60-min time point showed the desired size range and duplicate samples were prepared by the same method for each organism. Fragmentation of phage DNAs were performed using $1 \mu \mathrm{g}$ aliquots of PA1 and CRW10 in a $20 \mu \mathrm{l}$ reaction volume including fragmentation reaction buffer to $1 \times$, BSA, and $2 \mu \mathrm{l}$ NEB fragmentase enzyme (NEB). The reaction was incubated on ice for $5 \mathrm{~min}$ followed by incubation at $37^{\circ} \mathrm{C}$ for $20 \mathrm{~min}$ and stopped with $5 \mu \mathrm{l} 500 \mathrm{mM}$ EDTA and cleaned using MinElute columns (Qiagen).

\section{Post-fragmentation library preparation}

Post-fragmentation library preparation on the duplicate samples of E. coli CC118 sonication and fragmentase (60 min), and $H$. sapiens NA18507 fragmentase (60 min), was carried out as per standard Illumina methods, including a size selection using Novex TBE gels (Invitrogen), excising the 400-500 bp band. Final PCR amplification was carried out on a Bio-Rad (Hercules, CA, USA) MiniOpticon using SYBR Green I as a dye to monitor amplification. $1 \mu \mathrm{l}$ of each final library was run on a Novex TBE gel (Invitrogen) for library size confirmation. Nebulized or fragmentase-treated phage samples were size-selected using SPRI beads (Beckman Coulter, Danvers, MA, USA) and used to construct libraries according to standard protocols, including endpolishing, adaptor ligation, fill-in, and single-strand isolation. Adaptor sequences included multiplex identifiers (barcodes).

\section{Transposase-based library preparation}

Transposase-based library preparation for E. coli CC118 and H. sapiens NA18507 Illumina-compatible libraries used $50 \mathrm{ng}$ of genomic DNA brought up to $15 \mu \mathrm{l}$ in nuclease-free water (Ambion) followed by the addition of $4 \mu \mathrm{l} 5 \times$ LMW Nextera reaction buffer and $1 \mu \mathrm{l}$
Nextera enzyme mix (Illumina-compatible; Epicentre), followed by a gentle vortex and brief centrifugation. Each reaction tube was incubated at $55^{\circ} \mathrm{C}$ in a thermocycler with a heated lid for 5 min followed by placement on ice and immediate purification using a QIAquick PCR purification kit and elution in $20 \mu \mathrm{l}$ buffer EB (Qiagen). Suppression PCR was then carried out using $10 \mu \mathrm{l}$ of the eluate as template with $11.5 \mu \mathrm{l}$ nuclease-free water (Ambion), $25 \mu \mathrm{l} 2 \times$ Nextera PCR buffer, $0.5 \mu \mathrm{l}$ SYBR Green, $1 \mu \mathrm{l} 50 \times$ Nextera primer cocktail (Illumina-compatible), $1 \mu \mathrm{l} 50 \times$ Nextera adaptor 2 (barcodes 1-2 for E. coli and 3-4 for H. sapiens), and $1 \mu$ l Nextera PCR enzyme. The reaction was cycled in a Bio-Rad MiniOpticon to monitor the reaction under the following conditions: $(1 \times) 3: 00 \mathrm{~min}$ at $72^{\circ} \mathrm{C}$ and $(1 \times)$ 0:30 $\mathrm{min}$ at $95^{\circ} \mathrm{C}$, followed by 13 cycles of $\left[0: 10 \mathrm{~min}\right.$ at $95^{\circ} \mathrm{C}, 0: 30$ min at $62^{\circ} \mathrm{C}, 3: 00 \mathrm{~min}$ at $72^{\circ} \mathrm{C}$ ] for $E$. coli barcodes 1 and 2 and $H$. sapiens barcodes 3 and 4 (12 cycles for $H$. sapiens barcode 4 ). $1 \mu \mathrm{l}$ of each post-PCR library was electrophoresed through a Novex TBE gel (Invitrogen) for library size confirmation. Size selection of the E. coli CC118 transposase libraries was carried out at Epicentre Biotechnology using Agencourt AMPure (> 300 bp size selection), Zymo DNA purification (no size selection), or Caliper ( $350 \pm 10 \%$ bp size selection) methods. The $D$. melanogaster library was constructed by pooling two standard Nextera reactions following the manufacturer's protocol (Epicentre). For each reaction, 50 ng genomic DNA was initially tagmented (in vitro transposase-catalyzed adaptor insertion) at $55^{\circ} \mathrm{C}$ for $5 \mathrm{~min}$, and then MinElute purified. This was followed by PCR amplification with same conditions as with $H$. sapiens and $E$. coli libraries for 12 cycles. 400-450 bp gel-based size selection was carried out prior to sequencing.

A total of seven $H$. sapiens YH1 libraries were constructed, differing in mass of DNA, number of PCR cycles, and selected DNA fragment size. These included two (about $500 \mathrm{bp}$ and about $550 \mathrm{bp}$ ) produced from pooling five standard Nextera reactions, three (400$500 \mathrm{bp}, 500-550 \mathrm{bp}$ and 550-600 bp) produced from pooling two modified reactions with nine cycles of PCR enrichment, and another two libraries (300-500 bp and 500-650 bp) from a single tagmentation reaction using 500 ng starting DNA with five cycles of PCR enrichment. The insert-size distribution and final yields for the Drosophila and $H$. sapiens YH1 libraries were validated separately using a 2100 Bioanalyzer (DNA 1000 and 7500 kit; Agilent, Santa Clara, CA, USA) and quantitative PCR.

$P$. aeruginosa $\mathrm{PAO} 1$ Illumina-compatible shotgun libraries were prepared for each strain using Epicentre Biotechnologies' Nextera DNA sample preparation kits with a customized, unique 9 bp barcode sequence for each strain. The tagmentation reaction consisted of 
200 ng PAO1 DNA, $25 \mu$ l Nextera high molecular weight buffer, $1 \mu$ l Nextera transposase enzyme, and water to a total volume of $20 \mu \mathrm{l}$. The reaction was incubated for $5 \mathrm{~min}$ at $55^{\circ} \mathrm{C}$, cleaned using Qiagen MiniElute columns, and eluted in $11 \mu \mathrm{l}$ water. PCR reactions included $5 \mu \mathrm{l}$ of the fragmented DNA, $17 \mu \mathrm{l}$ water, $25 \mu \mathrm{l}$ Nextera PCR buffer, $1 \mu \mathrm{l}$ Nextera PCR enzyme, $1 \mu \mathrm{l}$ of a Nextera primer cocktail containing two short primers (at $10 \mu \mathrm{M}$ each) and one long Illumina-compatible adaptor (at $5 \mu \mathrm{M}$ ), and $1 \mu \mathrm{l}$ of the barcode containing Illumina adaptor (at $5 \mu \mathrm{M})$. PCR conditions used were the same as above using 12 cycles of amplification, followed by MinElute clean-up as before. Samples were run on a Novex TBE polyacrylamide gel to confirm library quality, and DNA concentrations measured using a Nanodrop.

For the Roche (454)-compatible libraries, standard Nextera reaction conditions were used with $50 \mathrm{ng}$ CRW10 (barcode 11) or PA1 (barcode 10) bacteriophage DNA, 454-Titanium compatible kit components and standard PCR methods, cycling 15 times. The PCR products were purified using Qiagen MinElute columns. Library fragment sizes were assessed using an Agilent Bioanalyzer DNA1000 chip.

\section{Targeted sequence capture of the human exome}

Libraries were prepared by transposase-catalyzed adaptor insertion by previously described methods using 50 ng genomic DNA (BK229.03, SFARI-SSC), $1 \mu \mathrm{l}$ transposomes, $4 \mu \mathrm{l} 5 \times \mathrm{HMW}$ buffer, and water to $20 \mu \mathrm{l}$. Samples were incubated at $55^{\circ} \mathrm{C}$ for $5 \mathrm{~min}$ then cleaned up (AMPure) and eluted in $20 \mu \mathrm{l}$ followed by the addition of $25 \mu \mathrm{l} 2 \times$ Nextera PCR buffer, $1 \mu \mathrm{l} 50 \times$ Nextera primer cocktail, $1 \mu \mathrm{l}$ Nextera PCR enzyme, $0.5 \mu \mathrm{l} 100 \times$ SYBR Green, and $1 \mu \mathrm{l}$ of a barcoded adaptor (Table S4 in Additional file 3) with water to $50 \mu$ l. Reactions were carried out on a Bio-Rad MiniOpticon using recommended cycling conditions for 12 rounds. Each tube was cleaned up (AMPure, Agencourt, Boston, MA, USA) and checked for size and quantity on an Agilent Bioanalyzer DNA 1000 chip. One sample was selected for capture using all of the $414.6 \mathrm{ng}$ for hybridization to Nimblegen (Madison, WI, USA) SeqCap EZ Exome probes v1.0 as per Nimblegen protocols using custom blockers (Nextera_Block1: 5'-AAT GAT ACG GCG ACC ACC GAG ATC TAC ACG CCT CCC TCG CGC CAT CAG AGA TGT GTA TAA GAG ACA G-3', Nextera_Block1_REV: 5'-CTG TCT CTT ATA CAC ATC TCT GAT GGC GCG AGG GAG GCG TGT AGA TCT CGG TGG TCG CCG TAT CAT T-3', Nextera_Block2: 5'-CAA GCA GAA GAC GGC ATA CGA GAT CGG TCT GCC TTG CCA GCC CGC TCA GAG ATG TGT ATA AGA GAC AG-3', Nextera_Block2_REV: 5'CTG TCT CTT ATA CAC ATC TCT GAG CGG GCT
GGC AAG GCA GAC CGA TCT CGT ATG CCG TCT TCT GCT TG-3') for $72 \mathrm{~h}$ at $47^{\circ} \mathrm{C}$. After hybridization, wash was performed as per Nimblegen protocols with streptavidin-coupled magnetic beads. Finally, PCR amplification was performed on exome captured library (Post_Cap_Short_For_Amp: 5'-AAT GAT ACG GCG ACC ACC GAG ATC T-3', Post_Cap_Short_Rev_Amp: 5'-CAA GCA GAA GAC GGC ATA CGA GAT-3'; 1× [0:30 min at $\left.98^{\circ} \mathrm{C}\right], 17 \times\left[0: 10 \mathrm{~min}\right.$ at $98^{\circ} \mathrm{C}, 0: 30 \mathrm{~min}$ at $65^{\circ} \mathrm{C}, 0: 45 \mathrm{~min}$ at $\left.72^{\circ} \mathrm{C}\right]$ ) followed by clean up (AMPure) and sequencing on an Illumina GAIIx SE36 run.

\section{PCR-free library preparation}

Adaptor sequences (NoPCR1: 5'-AAT GAT ACG GCG ACC ACC GAG ATC TAC ACG CCT CCC TCG CGC CAT CAG AGA TGT GTA TAA GAG ACA G-3', and NoPCR2: 5'-CAA GCA GAA GAC GGC ATA CGA GAT CGG TCT GCC TTG CCA GCC CGC TCA GAG ATG TGT ATA AGA GAC AG-3') were designed to contain the original 'Nextera' adaptor sequences, but with an additional 5' overhang of either P1 or P2 on adaptor 1 or adaptor 2, respectively (i.e. sequences to make compatible with cluster PCR on Illumina flowcell), thus eliminating the need to add them during a PCR step. The 5 ' phosphorylated reverse compliment of the 19 bp mosaic end (ME: 5'-Phos-CTG TCT CTT ATA CAC ATC T-3') sequence was hybridized to NoPCR $1 / 2$ by combining $5 \mu \mathrm{l}$ of each NoPCR 1 and NoPCR2 with $10 \mu \mathrm{l}$ ME reverse complement all at 100 $\mu \mathrm{M}$ with $80 \mu \mathrm{l} \mathrm{TE}$, followed by denaturation at $95^{\circ} \mathrm{C}$ for $5 \mathrm{~min}$ then slow cooling to room temperature for a final annealed adaptor concentration of $10 \mu \mathrm{M}$. Transposomes were assembled by incubating $5 \mu \mathrm{l}$ annealed adaptors at $10 \mu \mathrm{M}$ with $5 \mu \mathrm{l} 100 \%$ glycerol and $10 \mu \mathrm{l}$ $\mathrm{Ez}-\mathrm{Tn} 5$ transposase (Epicentre) and allowed to incubate at room temperature for $20 \mathrm{~min}$.

Tagmentation was carried out using previously prepared E. coli (CC118) or human (NA18507) genomic DNA using either 100 or $200 \mathrm{ng}$ of DNA, $5 \mu$ l prepared transposomes, $2 \mu \mathrm{l} 5 \times$ Nextera HMW buffer (Epicentre), and water to $10 \mu \mathrm{l}$. Reactions were incubated at $55^{\circ} \mathrm{C}$ for $5 \mathrm{~min}$, followed by the addition of $25 \mu \mathrm{l} 2 \times$ FailSafe PCR master mix (Epicentre), $1 \mu \mathrm{l}$ FailSafe DNA polymerase (Epicentre), and $14 \mu \mathrm{l}$ nuclease-free water (Ambion), and subsequent $5 \mathrm{~min}$ incubation at $72^{\circ} \mathrm{C}$ for nick translation. Tubes were then cleaned up using Qiaquick MinElute PCR purification columns (Qiagen), eluting in $12 \mu \mathrm{l}$ buffer EB.

For each reaction, $2 \mu \mathrm{l}$ was used as template for a real time PCR on a MiniOpticon (Bio-Rad) using $0.5 \mu \mathrm{l}$ SYBR Green, $25 \mu \mathrm{l} 2 \times$ Nextera PCR master mix, $1 \mu \mathrm{l}$ Nextera PCR enzyme and nuclease-free water to $50 \mu \mathrm{l}$. Alongside the NoPCR reactions, libraries of known concentrations were used as template at successive dilutions 
to be used as a standard for rough library quantification. Standard Nextera cycling conditions were used, without the initial $72^{\circ} \mathrm{C}$ extension step. PCR reactions were cleaned up by Qiaquick PCR purification columns and run on a Noved 6\% TBE PAGE gel (Invitrogen) for library size verification. After quantification, libraries were sequenced as per standard Illumina GAIIx protocol as a paired-end $36 \mathrm{bp}$ run.

\section{Low input transposase-based library preparation}

For the $500 \mathrm{pg}$ and $100 \mathrm{pg}$ E. coli (CC118) libraries and 10 pg human library (NA18507, Coriell), genomic DNA (in $1 \mu$ l volume) was incubated with $1 \mu$ l Nextera Illumina-compatible transposomes (Epicentre) at a 1 to 50 dilution $(1 \mu \mathrm{l}$ Nextera enzyme, $24 \mu \mathrm{l}$ TE, $25 \mu \mathrm{l}$ 100\% glycerol), $1 \mu \mathrm{l} 5 \times$ Nextera HMW buffer, and $2 \mu$ l nucleasefree water (Ambion). To avoid contamination, all dilutions and reaction preparation was carried out in a PCR hood. Reactions were incubated at $55^{\circ} \mathrm{C}$ for $5 \mathrm{~min}$ followed by addition of $25 \mu \mathrm{l} 2 \times$ Nextera PCR buffer, $0.5 \mu \mathrm{l}$ SYBR Green, $1 \mu \mathrm{l} 50 \times$ Nextera primer cocktail, and $1 \mu \mathrm{l} 0.5 \mu \mathrm{M}$ barcode adaptor 2 (barcodes A6, A9, or A4 for $500 \mathrm{pg}$ and $100 \mathrm{pg}$ E. coli DNA, or $10 \mathrm{pg}$ human DNA, respectively) and cycled under standard Nextera conditions in a MiniOpticon (Bio-Rad) real-time PCR thermocycler. Both reactions were removed after 20 cycles and cleaned up using Qiaquick MinElute columns, eluting in $20 \mu \mathrm{l} \mathrm{EB}$. Libraries were run on a $6 \%$ Novex TBE PAGE gel (Invitrogen) for size verification and sequenced as barcoded spike-ins as per standard Illumina GAIIx protocol as a paired-end $101 \mathrm{bp}$ (plus 9 bp barcode) run for $E$. coli libraries and a paired-end $36 \mathrm{bp}$ (plus $9 \mathrm{bp}$ barcode) run for human.

\section{Direct colony-based library preparation}

Fusion-Blue chemically competent E. coli (Clontech, Mountain View, CA, USA) were transformed with pUC19 bearing a $2 \mathrm{kbp}$ insert of human genomic DNA, and then plated on Luria broth (LB) + ampicillin. A small number of cells were picked from a single bacterial colony with a $10 \mu$ l pipette tip, and then transferred with dipping into $15 \mu \mathrm{l}$ nuclease-free $\mathrm{H}_{2} \mathrm{O}$. The suspended cells were heat-lysed at $95^{\circ} \mathrm{C}$ for $5 \mathrm{~min}$, then placed on ice for $2 \mathrm{~min}$. Nextera $5 \times$ LMW reaction buffer and enzyme (Illumina-compatible; Epicentre) were added to the sample, followed by brief mixing and incubation at $55^{\circ} \mathrm{C}$ for $5 \mathrm{~min}$. The reaction was then stopped by heating to $70^{\circ} \mathrm{C}$ for $15 \mathrm{~min}$. Sequencing-compatible primer sites were added in a $50 \mu \mathrm{l}$ PCR reaction using $5 \mu \mathrm{l}$ of the transposase reaction directly as template without intervening purification. PCR was carried out with $31.6 \mu \mathrm{H}_{2} \mathrm{O}, 10 \mu \mathrm{l}$ Kapa $2 \mathrm{G}$ robust A buffer $5 \times$ (Kapa Biosystems, Cape
Town, South Africa), $1 \mu \mathrm{d}$ dNT mix (10 mM each), $0.25 \mu \mathrm{l}$ SYBR Green $100 \times, 1 \mu \mathrm{l} 50 \times$ Nextera primer cocktail, $1 \mu \mathrm{l} 50 \times$ Nextera adaptor 2 , and $0.20 \mu \mathrm{l} \mathrm{Kapa}$ $2 \mathrm{G}$ robust polymerase; cycling conditions were as described by Epicentre. The amplification reaction was cleaned up with a Qiaquick PCR clean-up column (Qiagen) and eluted into $50 \mu \mathrm{LB}$.

\section{Sequencing}

Sequencing of the H. sapiens NA18507, E. coli CC118, and $D$. melanogaster libraries was done on an Illumina Genome Analyzer IIx as paired-end 36, 36, and 45 bp runs, respectively, using standard read primers for sonication and fragmentase libraries, run in individual lanes, and Nextera read primers for Nextera libraries. $H$. sapiens $\mathrm{YH} 1$ libraries were run on an Illumina HiSeq2000 as paired-end 90 bp run using Nextera read primers. Phage libraries contained library-specific barcodes and were run as multiplexed samples using GS FLX Titanium sequencing protocols. P. aeruginosa libraries were pooled by combining $100 \mathrm{ng}$ of each strain library, and sequenced on an Illumina Genome Analyzer IIx with a paired-end 76-cycle run.

\section{Short read mapping}

Short read mapping was done on the E. coli CC118, D. melanogaster and H. sapiens (NA18507 \& YH1) Illumina GAIIx or HiSeq2000 sequenced samples by converting the raw sequence files to fastq format and then mapping to the hg18 (NCBI36) reference using the BWA [16] alignment software. After mapping, PCR duplicates were removed, as well as read-pairs with an insert size shorter than that of the read length.

\section{Long read assembly}

Long read assembly from bacteriophage samples sequenced on the Roche GS FLX Ti was done using Roche's newbler assembler under default parameters. Individual reads from each dataset were mapped against the assembled genome using gsMapper (Roche Software Release: 2.3 (091027_1459)).

\section{Fragmentation site characterization}

Fragmentation site characterization was carried out by stacking all regions of the genome flanking forward strand mapping start locations and the reverse complement of reverse strand start locations followed by calculating nucleotide frequencies at each position relative to the fragmentation site, thus generating a positional weight matrix (PWM). The PWMs then were imported into the SeqLogo (Oliver Bembom, Dept. of Biostatistics, University of California, Berkeley, 2008) package for Bioconductor in $\mathrm{R}$ and used to generate positional 
information content (IC) and sequence logos using the equation outlined by T. D. Schneider et al. [21,22]:

$$
\mathrm{IC}(\mathrm{w})=\log _{2}(\mathrm{~J})+\sum_{j=1}^{\mathrm{J}} p_{w j} \log _{2}\left(p_{w j}\right)=\log _{2}(\mathrm{~J})-\operatorname{entropy}(w)
$$

where $J$ is the number of variables in the alphabet (4; $\mathrm{A}, \mathrm{C}, \mathrm{G}$, or $\mathrm{T}$ ), and $j$ is the base at position $w$. This equation does not factor in the background nucleotide frequencies.

\section{Normalization of Illumina GAllx coverage}

Normalization of Illumina GAIIx coverage for the E. coli (non-size-selected) data was done by dividing the coverage at each position in the genome by the total number of mapped bases and then multiplying by a constant (the average number of mapped bases was close to $1 \mathrm{~Gb}$, therefore $10^{9}$ was used as the constant).

\section{Coverage distribution histograms}

Coverage distribution histograms were generated by calculating the number of times each base of the genome was sequenced and plotting the frequency of each level of coverage.

\section{Coverage by $\mathrm{G}+\mathrm{C}$ content}

Coverage by $\mathrm{G}+\mathrm{C}$ content plots were generated by binning the reference genome into $500 \mathrm{bp}$ bins for E. coli, $10 \mathrm{kbp}$ bins for human, and $1 \mathrm{kbp}$ bins for Drosophila (other sizes were also investigated, resulting in very similar distributions) and calculating the $\mathrm{G}+\mathrm{C}$ content of each bin, followed by plotting the coverage of that bin.

\section{Library complexity}

Library complexity was calculated by random sampling of 50,000 read-pairs without replacement and plotting the number of uniquely occurring read-pairs versus the total number of sampled read-pairs.

\section{Insertion size distributions}

Insertion size distributions were generated by taking the distance between the start mapping location of the first read and the end mapping location of the second read for every read-pair and plotting the frequency of occurrences of each insert size.

\section{SNP calls}

SNP calls for the YH1 genome were generated using the SAMtools [18] variant caller with a maximum coverage of 1,000 and minimum quality score of 30 . Prior to variant calling, read-pairs with an insert size less than $90 \mathrm{bp}$ and reads not properly paired were removed to reduce noise. Calls were then compared with the SNPs reported by Wang et al. (2008) [15] and to dbSNP build 129.

\section{Cell-line-discordant SNP calls}

In order to minimize false calls due to mapping errors, a repeat-masked version of hg18 was used along with further masking with respect to mappability according to the UCSC Genome Browser 'Rosetta 35mer uniqueness' and excluding all regions with a score of 0 (this score means that the sequence maps perfectly to multiple locations in the genome). This track was used because it was generated using the BWA aligner that was used in our analysis, and because the original YH1 sequence data is made up of $36 \mathrm{bp}$ reads. Out of this newly masked genome, positions were called that had a SNP quality score in the cell line over 30, a reference call in blood over 30 , and coverage less than $100 \times$ in both datasets. Of those with a quality score of 50 in both for their calls, 100 were randomly chosen for validation.

Validation was carried out using a mass spectrometry assay (Sequenom, San Diego, CA, USA). Primers for PCR amplification and extension were successfully designed for 100 mutation sites using the Sequenom MassArray Assay Design v3.1. PCR amplification, shrimp alkaline phosphatase treatment of unincorporated dNTPs, probe extension and resin desalting were carried out in sequence using the conditions described elsewhere [23]. Sequenom genotyping was performed in parallel for genomic DNA from YH1 blood and the same batch of lymphoblastoid cell lines as was used for sequencing. A negative control and technical replicate were also run in parallel for each typed position. Genotyping of all 100 testing sites passed the filter criteria of: (1) no failing extension, (2) no false positive in the negative control, (3) consistency between two technical replicates. Genotyping was further performed for the 100 positions in the $\mathrm{YH}$ primary lymphoblastoid cell lines using the same method, with 98 meeting the above filter criteria.

\section{Exome analysis}

Sequence reads from transposase-based libraries subjected to human exome capture were aligned to the human reference (hg18) using BWA [16]. Each aligned base was deemed to be on target if it was within 100 bases of a targeted sequence. At each position within target regions, coverage was assessed and any position with a depth of at least one was considered covered. Comparison to standard exome methods was made by trimming read 1 of a PE76 lane from a GAIIx down to 36 bases and aligning it the same way. Because the standard library had fewer reads mapped, 28 million reads were randomly taken from both libraries and above analysis performed. Complexity was interrogated by taking an equivalent number of on-target SE36 reads generated 
by each method and calculating the percentage with unique start-points.

\section{Barcode design}

Barcode design yielded a set of $96 \times 9$ bp sequences in which each $9 \mathrm{bp}$ sequence contained no homopolymer run of three or more bases, had a GC content of $\leq 60 \%$, was a edit distance of at least four away from all other members of the set of 96, and screened negative when compared with other adaptor and primer sequences used here. Also, we took care to ensure that each base (A, G, $\mathrm{C}$, or $\mathrm{T}$ ) was represented at least once in each $9 \mathrm{bp}$ barcode, and at least once in each position along the $9 \mathrm{bp}$.

\section{Barcode deconvolution}

Barcode deconvolution for pooled, multiplexed Pseudomonas samples was carried out by computing the Levenshtein edit distance between the obtained index read and each of the 96 barcode sequences used. The corresponding read-pair was assigned to a barcode when that barcode was within edit distance of 2 of the index read, with the next closest matching barcode being at least two further edits away.

\section{Data accessibility}

All sequence data described here is being deposited in the NCBI Sequence Read Archive (SRA) under accession SRP004087.

\section{Additional material}

Additional file 1: Supplementary Tables 1-3.

Additional file 2: Supplementary Figures 1-8.

Additional file 3: Supplementary Table 4. S4: barcode adaptors.

\begin{abstract}
Acknowledgements
We would like to acknowledge W Reznikoff for conceptual contributions; H Grunenwald and others at Epicentre for early access to materials; C Lee, J Hiatt, R Patwardhan, L Felker, R Qiu, B O'Roak, and other members of the Shendure Lab for assistance with protocol development and sequencing; the genome sequencing platform of BGI-Shenzhen, especially Wu Kui, Jiang Hui, Chen Mingfeng, Liu Lin, Yu Lili and Kaileung Yuan, for their contributions to YH1 and Drosophila genome sequencing; He Weiming, Liu Xingwang and Yang Lei for their genotyping work; and I. Meek and E. Wong-Ho (Caliper) for size selections on the LabChip XT. This work was supported in part by grants from the National Institutes of Health/National Heart Lung and Blood Institute (R01 HL094976 to JS), the National Institutes of Health/National Human Genome Research Institute (R21 HG004749 to JS), the National Institutes of Health/National Institute of Allergy and Infectious Disease Northwest Regional Center of Excellence for Biodefense and Emerging Infectious Diseases at the University of Washington (3U54AI05714), the Ministry of Science and Technology of China, 863 program (2006AA02A301), and an NSF Graduate Research Fellowship (to JOK).

\section{Author details}

'Department of Genome Sciences, University of Washington, Seattle, WA 98195, USA. ${ }^{2}$ The Marine Biological Laboratory, Woods Hole, MA 02543, USA.
\end{abstract}

${ }^{3}$ BGl-Shenzhen, Shenzhen 518000, China. ${ }^{4}$ Epicentre Biotechnologies, 726 Post Road, Madison, WI 53713, USA.

\section{Authors' contributions}

AA, HGM, Asan, XX, NCC, XZ, and JS conceived the study and planned experiments. $A A$ analyzed all data, conducted comparison experiments with the Illumina platform, and developed the PCR-free protocol, the exome capture protocol, and the low-input protocol. HGM conducted comparison experiments and accompanying analyses with the 454 platform. Asan, XX, and XZ performed $\mathrm{YH} 1$ and Drosophila genome sequencing, and SNP validation. JOK developed the direct-from-colony library construction protocol and analyzed the resulting data. APM performed exome capture experiments. EHT, BS and AA developed and tested the 96-plex barcoding protocol. AA and JS wrote the manuscript. All authors reviewed drafts, contributed comments, and approved the final manuscript.

\section{Competing interests}

NCC is an employee of Epicentre Biotechnologies. The other authors declare that they have no completing interests.

Received: 22 September 2010 Accepted: 8 December 2010 Published: 8 December 2010

\section{References}

1. Kahvejian A, Quackenbush J, Thompson JF: What would you do if you could sequence everything? Nat Biotechnol 2008, 26:1125-1133.

2. Shendure J, Ji H: Next-generation DNA sequencing. Nat Biotechnol 2008, 26:1135-1145.

3. Bentley DR, Balasubramanian S, Swerdlow HP, Smith GP, Milton J, Brown CG, Hall KP, Evers DJ, Barnes CL, Bignell HR, Boutell JM, Bryant J, Carter RJ, Keira Cheetham R, Cox AJ, Ellis DJ, Flatbush MR, Gormley NA, Humphray SJ, Irving L, Karbelashvili MS, Kirk SM, Li H, Liu X, Maisinger KS, Murray $L$, Obradovic B, Ost T, Parkinson ML, Pratt MR, et al: Accurate whole human genome sequencing using reversible terminator chemistry. Nature 2008, 456:53-59.

4. Margulies M, Egholm M, Altman WE, Attiya S, Bader JS, Bemben LA, Berka J, Braverman MS, Chen YJ, Chen Z, Dewell SB, Du L, Fierro JM, Gomes XV, Godwin BC, He W, Helgesen S, Ho CH, Irzyk GP, Jando SC, Alenquer ML, Jarvie TP, Jirage KB, Kim JB, Knight JR, Lanza JR, Leamon JH, Lefkowitz SM, Lei M, Li J, et al: Genome sequencing in microfabricated high-density picolitre reactors. Nature 2005, 437:376-380.

5. McKernan KJ, Peckham HE, Costa GL, McLaughlin SF, Fu Y, Tsung EF, Clouser CR, Duncan C, Ichikawa JK, Lee CC, Zhang Z, Ranade SS, Dimalanta ET, Hyland FC, Sokolsky TD, Zhang L, Sheridan A, Fu H, Hendrickson CL, Li B, Kotler L, Stuart JR, Malek JA, Manning JM, Antipova AA, Perez DS, Moore MP, Hayashibara KC, Lyons MR, Beaudoin RE, et al: Sequence and structural variation in a human genome uncovered by short-read, massively parallel ligation sequencing using two-base encoding. Genome Res 2009, 19:1527-1541.

6. Eid J, Fehr A, Gray J, Luong K, Lyle J, Otto G, Peluso P, Rank D, Baybayan P, Bettman B, Bibillo A, Bjornson K, Chaudhuri B, Christians F, Cicero R, Clark S, Dalal R, Dewinter A, Dixon J, Foquet M, Gaertner A, Hardenbol P, Heiner C, Hester K, Holden D, Kearns G, Kong X, Kuse R, Lacroix Y, Lin S, et al: Realtime DNA sequencing from single polymerase molecules. Science 2009, 323:133-138.

7. Mortazavi A, Williams BA, McCue K, Schaeffer L, Wold B: Mapping and quantifying mammalian transcriptomes by RNA-Seq. Nat Methods 2008 , 5:621-628.

8. Lennon NJ, Lintner RE, Anderson S, Alvarez P, Barry A, Brockman W, Daza R, Erlich RL, Giannoukos G, Green L, Hollinger A, Hoover CA, Jaffe DB, Juhn F, McCarthy D, Perrin D, Ponchner K, Powers TL, Rizzolo K, Robbins D, Ryan E, Russ C, Sparrow T, Stalker J, Steelman S, Weiand M, Zimmer A, Henn MR, Nusbaum C, Nicol R: A scalable, fully automated process for construction of sequence-ready barcoded libraries for 454. Genome Biol 11:R15.

9. Craig DW, Pearson JV, Szelinger S, Sekar A, Redman M, Corneveaux JJ, Pawlowski TL, Laub T, Nunn G, Stephan DA, Homer N, Huentelman MJ: Identification of genetic variants using bar-coded multiplexed sequencing. Nat Methods 2008, 5:887-893.

10. Quail MA, Kozarewa I, Smith F, Scally A, Stephens PJ, Durbin R, Swerdlow H, Turner DJ: A large genome center's improvements to the Illumina sequencing system. Nat Methods 2008, 5:1005-1010. 
11. Kozarewa I, Ning Z, Quail MA, Sanders MJ, Berriman M, Turner DJ: Amplification-free Illumina sequencing-library preparation facilitates improved mapping and assembly of $(\mathrm{G}+\mathrm{C})$-biased genomes. Nat Methods 2009, 6:291-295.

12. Mamanova L, Andrews RM, James KD, Sheridan EM, Ellis PD, Langford CF, Ost TW, Collins JE, Turner DJ: FRT-seq: amplification-free, strand-specific transcriptome sequencing. Nat Methods 2010, 7:130-132.

13. Reznikoff WS: Tn5 as a model for understanding DNA transposition. Mol Microbiol 2003, 47:1199-1206.

14. Reznikoff WS: Transposon Tn5. Annu Rev Genet 2008, 42:269-286.

15. Wang J, Wang W, Li R, Li Y, Tian G, Goodman L, Fan W, Zhang J, Li J, Guo Y, Feng B, Li H, Lu Y, Fang X, Liang H, Du Z, Li D, Zhao Y, Hu Y, Yang Z, Zheng H, Hellmann I, Inouye M, Pool J, Yi X, Zhao J, Duan J, Zhou Y, Qin J, Ma L, et al: The diploid genome sequence of an Asian individual. Nature 2008, 456:60-65.

16. Li H, Durbin R: Fast and accurate short read alignment with BurrowsWheeler transform. Bioinformatics 2009, 25:1754-1760.

17. Goryshin IY, Miller JA, Kil YV, Lanzov VA, Reznikoff WS: Tn5/IS50 target recognition. Proc Natl Acad Sci USA 1998, 95:10716-10721.

18. Li H, Handsaker B, Wysoker A, Fennell T, Ruan J, Homer N, Marth G, Abecasis G, Durbin R: The Sequence Alignment/Map format and SAMtools. Bioinformatics 2009, 25:2078-2079.

19. Manoil C, Beckwith J: TnphoA: a transposon probe for protein export signals. Proc Natl Acad Sci USA 1985, 82:8129-8133.

20. Blin N, Stafford DW: A general method for isolation of high molecular weight DNA from eukaryotes. Nucleic Acids Res 1976, 3:2303-2308.

21. Schneider TD, Stormo GD, Gold L, Ehrenfeucht A: Information content of binding sites on nucleotide sequences. J Mol Biol 1986, 188:415-431.

22. Schneider TD, Stephens RM: Sequence logos: a new way to display consensus sequences. Nucleic Acids Res 1990, 18:6097-6100.

23. Yi X, Liang Y, Huerta-Sanchez E, Jin X, Cuo ZX, Pool JE, Xu X, Jiang H, Vinckenbosch N, Korneliussen TS, Zheng H, Liu T, He W, Li K, Luo R, Nie X, Wu H, Zhao M, Cao H, Zou J, Shan Y, Li S, Yang Q, Asan, Ni P, Tian G, Xu J, Liu $X$, Jiang T, Wu R, et al: Sequencing of 50 human exomes reveals adaptation to high altitude. Science 329:75-78.

doi:10.1186/gb-2010-11-12-r119

Cite this article as: Adey et al:: Rapid, low-input, low-bias construction of shotgun fragment libraries by high-density in vitro transposition. Genome Biology 2010 11:R119.

\section{Submit your next manuscript to BioMed Central and take full advantage of:}

- Convenient online submission

- Thorough peer review

- No space constraints or color figure charges

- Immediate publication on acceptance

- Inclusion in PubMed, CAS, Scopus and Google Scholar

- Research which is freely available for redistribution

Submit your manuscript at www.biomedcentral.com/submit
Biomed Central 